\title{
Towards Subject-Specific Models of the Dynamic Heart for Image-Guided Mitral Valve Surgery
}

\author{
Cristian A. Linte ${ }^{1,2}$, Marcin Wierzbicki ${ }^{2}$, John Moore ${ }^{2}$, Stephen H. Little ${ }^{3,4}$, \\ Gérard M. Guiraudon ${ }^{1,4}$, and Terry M. Peters ${ }^{1,2}$ \\ ${ }^{1}$ Biomedical Engineering Graduate Program, University of Western Ontario \\ ${ }^{2}$ Imaging Research Laboratories, Robarts Research Institute \\ ${ }^{3}$ Division of Cardiology, University of Western Ontario \\ ${ }^{4}$ Canadian Surgical Technologies \& Advanced Robotics \\ London ON Canada \\ \{clinte, mwierz, jmoore, tpeters\} @imaging.robarts.ca
}

\begin{abstract}
Surgeons need a robust interventional system capable of providing reliable, real-time information regarding the position and orientation of the surgical targets and tools to compensate for the lack of direct vision and to enhance manipulation of intracardiac targets during minimally-invasive, off-pump cardiac interventions. In this paper, we describe a novel method for creating dynamic, pre-operative, subjectspecific cardiac models containing the surgical targets and surrounding anatomy, and how they are used to augment the intra-operative virtual environment for guidance of valvular interventions. The accuracy of these pre-operative models was established by comparing the target registration error between the mitral valve annulus characterized in the pre-operative images and their equivalent structures manually extracted from 3D US data. On average, the mitral valve annulus was extracted with a $3.1 \mathrm{~mm}$ error across all cardiac phases. In addition, we also propose a method for registering the pre-operative models into the intra-operative virtual environment.
\end{abstract}

\section{Introduction}

Minimally-invasive cardiac procedures can potentially reduce complications arising from surgical interventions by minimizing the size of the incision required to access the heart, while employing medical imaging to visualize intracardiac targets without direct vision [12]. Although less invasive, these procedures often involve myocardial arrest and use of cardiopulmonary bypass, which contribute to patient morbidity [3]. To further reduce invasiveness, techniques have been developed to perform interventions on the beating-heart 44.5].

This paper focuses specifically on mitral valve surgery. As part of an ongoing project, we have developed a robust interventional system to allow the surgeon to safely access cardiac chambers in the beating heart [6, as well as to visualize and deliver therapy to the mitral valve without compromising the quality of the procedure. The navigation system integrates trans-esophageal echocardiography (TEE) for real-time intracardiac visualization, a virtual reality (VR)

N. Ayache, S. Ourselin, A. Maeder (Eds.): MICCAI 2007, Part II, LNCS 4792, pp. 94-101 2007.

(C) Springer-Verlag Berlin Heidelberg 2007 
environment [7] that augments the TEE with pre-operative subject images and geometric models, and surgical instruments tracked in real time using a magnetic tracking system (MTS) 8]. The guidance of beating-heart mitral valve replacement requires detailed information regarding the dynamic behaviour of the valve annulus and surrounding anatomy. This information is not clearly portrayed by the TEE images, as 2D ultrasound (US) images lack anatomical context and adequate representation of the surgical tools. Although 3D TEE US might become a potential future solution to this problem, its narrow field of view may impose further challenges in visualizing the tools and target in the same volume. To address these limitations, we include pre-operative, patient-specific models derived from MR images, which incorporate a dynamic representation of the gross cardiac anatomy (eg. myocardium) and surgical target (mitral valve annulus - MVA) within the intra-operative VR environment. As a result, the intra-operative TEE information can be interpreted within a rich, high-quality 3D context 9 for improved procedure planning and navigation of surgical tools, while on-target positioning and detailed manipulations are performed under realtime US guidance.

To better illustrate our research goals, we follow the patient through the proposed mitral valve procedure work-flow. First, pre-operative cine MR images of the subject are acquired. During procedure planning, a prior high-quality heart model containing the MVA and surrounding anatomy is registered to the subjectspecific image dataset, to obtain a pre-operative, subject-specific cardiac model. After accessing the heart, the intra-operative MVA is defined interactively using tracked 2D TEE, and displayed within the intra-operative subject space. To facilitate tool navigation and improve spatial orientation, the 2D US images are complemented by 3D anatomical models obtained from pre-operative MR images, and combined with virtual representations of the tracked surgical tools. The pre-operative cardiac models are incorporated within the intraoperative space by aligning the pre-operative and intra-operative annuli using registration.

\section{Methodology}

Our objective was to assesses the accuracy of integrating pre-operative, subjectspecific cardiac models as part of the clinical routine. We used a prior highresolution heart model constructed from multiple-subject 4D MRI datasets [10] to segment the surgical target (MVA) and other relevant cardiac anatomy, by registering the prior model to a subject-specific pre-operative MR image dataset. Note that this latter image dataset was not employed in the construction of the prior model. The resulting subject-specific MVA was then compared to its true location, obtained by manual segmentation of 3D full-volume US images acquired throughout the cardiac cycle. The accuracy with which the MVA could be identified using the prior model was assessed by quantifying the target registration error (TRE) between the model-predicted annuli, and those extracted manually from US images. 


\subsection{Prior High-Resolution Heart Model}

MRI is often considered the gold-standard modality for cardiac imaging, given its superior soft tissue contrast and $4 \mathrm{D}$ imaging capabilities. However, clinical MR images may exhibit low spatial resolution, low signal-to-noise ratio (SNR), and motion artifacts. Consequently, surgical targets extracted directly from these clinical images may not be sufficiently accurate for the planning and guidance of the proposed mitral valve surgery. To address this concern, we used a high-quality prior heart model to characterize the surgical targets in the low-quality subject images. This model consisted of image and geometry components, and was built from low-resolution MR images of 10 subjects (6 mm slice thickness). Various anatomical features (i.e. left ventricular myocardium, right ventricle and atrium, etc.) were manually segmented from each image and the resulting data was then co-registered into a common high-resolution reference image $(1.5 \mathrm{~mm}$ slice thickness) [11. The image component of the prior model represents a measure of the MR appearance of the heart, and was obtained by performing a principal component analysis on the co-registered data. The geometry component consists of the previously described anatomical features segmented in the reference image, and corrected to fit the average shape of the population [10].

The prior model is used to segment anatomical features from clinical quality MR data, by fitting the image and geometry components simultaneously to a subject-specific dynamic image dataset. A similar approach was undertaken by Lorenzo-Valdès et al. [12, who constructed and segmented an average heart model based on population images, and registered it to target images to automate segmentation. Our final models specific to the left ventricular myocardium (LV), left atrium (LA) and right atrium and ventricle (RAV) were previously shown to be accurate within $5.4 \pm 0.8 \mathrm{~mm}$ [10, despite the low resolution of the subject data. For this work, we expanded the prior model by including the MVA as part of its geometry (Fig. 19a).

\subsection{Image Acquisition}

MR Imaging. Clinically feasible coronal images of one healthy subject were acquired using a 1.5T CVi scanner (GE Medical Systems, Milwaukee, USA). The imaging protocol employed an ECG-gated gradient echo pulse sequence, a $256 \times 128$ image matrix, two signal averages (NEX), $20^{\circ}$ flip angle, $7.6 \mathrm{~ms}$ TR, and $4.2 \mathrm{~ms}$ TE. The dataset consisted of $203 \mathrm{D}$ images throughout the cardiac cycle, with an in-plane resolution of $1.5 \times 1.5 \mathrm{~mm}^{2}, 6.0 \mathrm{~mm}$ slice thickness. To minimize breathing artifacts, 20 sec breath-holds were employed during the acquisition of each slice, for a total scan duration of $\sim 20 \mathrm{~min}$.

US Imaging. 3D US images of the same subject were acquired throughout the cardiac cycle on a Philips SONOS 7500 scanner. Full-volume apical images of the heart were acquired with a $19 \mathrm{~Hz}$ frame-rate and a $14 \mathrm{~cm}$ depth-of-focus, with the subject in the left lateral decubitus position. Breath-holds of $5-10 \mathrm{sec}$ were employed to minimize artifacts due to respiratory motion. 


\subsection{Anatomical Feature Extraction}

MR Image Segmentation. In addition to the anatomical features already present, we augmented the prior model introduced in section $\mathbf{2 . 1}$ with a representation of our surgical target (MVA). The annulus was segmented manually under the assistance of an experienced cardiologist, by interactively selecting points on the 3D image of the prior model depicting the heart at mid-diastole (MD). We employed a custom-developed spline-based segmentation tool similar to that available for clinical application within the TomTec 4D MV Assessment Software (Unterschleissheim, Germany). The new prior model consisted of a high-quality image component, gross cardiac anatomy, and MVA (Fig. 1a).
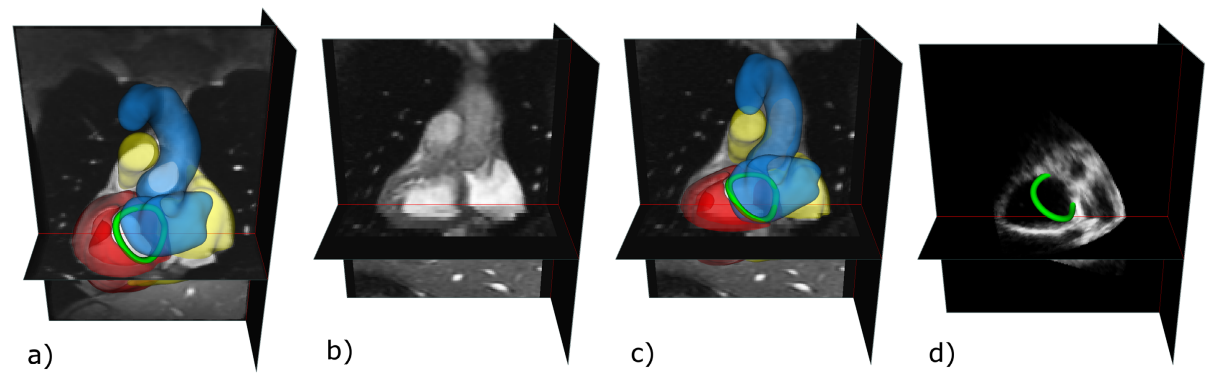

Fig. 1. a) Prior high-resolution cardiac model at MD, containing segmented LV, LA, RAV, and MVA; b) Clinical quality subject MR image at MD; c) Clinical quality subject MR image segmented using the prior model; d) Subject US image at MD showing the manually segmented MVA

This model was then registered to the low-quality, mid-diastole MR image of the subject (Fig. 1 b). The initial model-to-subject registration was performed using an affine transformation, which was then refined using a non-rigid transformation, to account for the remaining morphological differences between the source and target images. Registration was achieved by maximizing the mutual information (MI) between the model and subject image, while ensuring the prior geometry remained consistent with user-selected points in the subject image [10.

The resulting subject-specific model at MD (Fig. 1 $\mathbf{c}$ ) was then animated over the cardiac cycle by non-rigidly registering the MD frame to the remaining frames in the $4 \mathrm{D}$ dataset, and using these transformations to deform the MD subject-specific model throughout the cardiac cycle [13].

US Image Segmentation. The segmentation of the 3D US volumes was performed manually under the guidance of an experienced echocardiographer. The same spline-based technique as for the MR model was employed to outline the MVA contour at various time points throughout the cardiac cycle (Fig. 1 d d). In addition, the LV geometry was manually segmented from the MD image, and used to drive the registration of US data to the MR image space. This latter 
US to MR transform consisted of aligning geometric features (i.e. left ventricular surface) predefined in both the source and target image using an affine transformation, followed by further refinement using a non-rigid transformation.

\section{Results}

Our main goal was to determine the accuracy of our model-based segmentation approach when predicting the location of dynamic surgical targets. The accuracy was assessed by computing the root-mean-squared (RMS) TRE between the model-based MVA, and the MVA extracted manually from 3D US images and registered to the MR image space. The TRE was quantified at four different time points in the cardiac cycle: end-diastole (ED), mid-systole (MS), end-systole (ES), and mid-diastole (MD). In addition, we also estimated the perimeter for both the model-extracted and gold-standard annuli at each of these cardiac frames. A summary of these results is presented in Table $\mathbf{1}$.

Table 1. Mitral valve annulus perimeter and TRE values of the model-predicted MVA geometry and the gold-standard MVA, quantified at four phases throughout the cardiac cycle, using motion information extracted from both MR and US image datasets

\begin{tabular}{|c||c|c|c|c|c|c|}
\hline \multirow{2}{*}{$\begin{array}{c}\text { Cardiac } \\
\text { Phase }\end{array}$} & \multicolumn{2}{c|}{ MR-extracted Motion } & \multicolumn{3}{c|}{ US-Extracted Motion } \\
\cline { 2 - 5 } & Mean Perimeter (mm) & TRE & Mean Perimeter (mm) & $\begin{array}{c}\text { TRE } \\
\text { (mm) }\end{array}$ \\
\cline { 2 - 6 } & Model & Gold-Std. (US) & (mm) & Model & Gold-Std. (US) & (mm) \\
\hline \hline ED & 119.9 & 111.4 & 2.6 & 114.4 & 111.4 & 2.6 \\
MS & 121.3 & 118.0 & 7.9 & 113.0 & 118.0 & 3.5 \\
ES & 118.5 & 117.4 & 10.5 & 113.5 & 117.4 & 3.3 \\
MD & 114.3 & 109.7 & 2.9 & 114.3 & 109.7 & 2.9 \\
\hline
\end{tabular}

According to these results, the perimeter of the model-based MVA was consistently within $4.8 \%$ of that extracted from 3D US images, throughout all cardiac frames. Moreover, it was also observed that the annulus perimeter did not significantly change throughout the cardiac cycle. Nevertheless, a poor TRE of the two annuli sets was observed during the systolic phases, when the dynamic pre-operative surgical targets were obtained using motion information extracted from the 4D MR image dataset. These inconsistencies occurred predominantly on the mitral-aortic valve boundary. The main motion observed in this region of the MVA was caused by the systolic thrust, which is physiologically counteracted by the tension generated within the chordae tendinae by the papillary muscles. However, due to the limited information provided in this region by the thick-slice MR data, these intricate MVA motion patterns could not be correctly reconstructed using the MR images. On the other hand, 3D US images possess a much higher resolution, especially in the valve region, allowing for a clear identification of the valve leaflets. Therefore, we also employed the 4D US images to extract valvular motion using the same nonrigid image registration approach. As a result, the systolic TRE was significantly improved (Table 1 and (Fig. 20) ). 

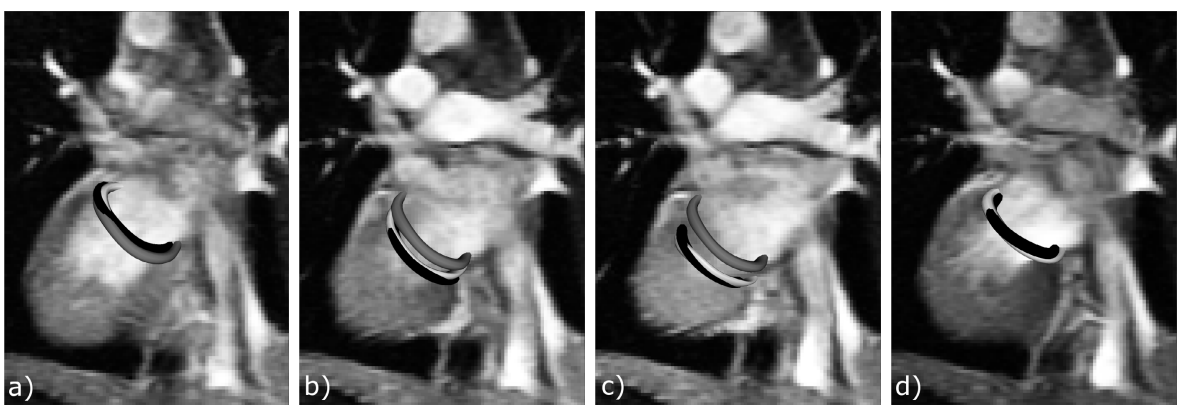

Fig. 2. Subject-specific MVA extracted manually from US images (black), model-based MVA animated using MR motion extraction (grey), and model-based MVA animated using US motion extraction (white). All annuli were registered to the MR space and displayed at four cardiac phases: a) ED; b) MS; c) ES; d) MD. Note how systolic inaccuracies caused by MR motion extraction (grey vs. black annuli) were significantly improved using US motion extraction (white vs. black annuli).

\section{Discussion}

This work constitutes the first steps in investigating the feasibility of employing pre-operative, subject-specific, dynamic models for enhancement of planning, and navigation of valvular procedures using a VR environment. Specifically, we determined the location of the surgical targets predicted by the pre-operative, subject-specific, dynamic models to be accurate within $3.1 \mathrm{~mm}$ with respect to their gold-standard location identified from 3D US images. This study clearly identified weaknesses regarding the extraction of accurate valvular motion patterns from clinically feasible MR images, and highlighted the need to acquire a set of full-volume 3D US images of the subject, to assist in building accurate pre-operative dynamic heart models.

Our results were successful, despite the small variations in anatomical structures identified in the MR and US images, as well as any subject-specific physiological variations between the times at which the images were acquired. These cardiac models will be used to augment the surgical virtual environment during image-guided mitral valve interventions. While enhancing procedure visualization by complementing the intra-operative space with $3 \mathrm{D}$ anatomical context, these models constitute a significant navigation aid. According to our collaborating cardiac surgeon, a misalignment on the order of approximately $5 \mathrm{~mm}$ is tolerable, as these models will be used to facilitate the navigation of instruments towards the surgical targets, whereas on-target positioning and fine tuning will be performed under real-time US guidance. In addition, the relative accuracy of the tracked surgical tools is on the order of 1-2 mm [14, leading to an accurate virtual tool to US navigation.

To demonstrate the usefulness of the pre-operative models, we performed a preliminary experiment in which we used these results to augment the intraoperative VR environment (Fig. 3). The registration consisted of aligning the 

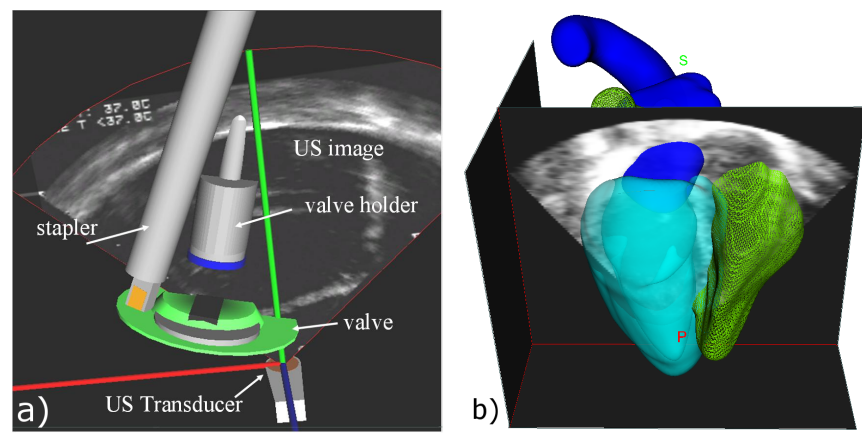

Fig. 3. a) Surgical VR environment consisting of 2D US probe and image, valveguiding tool, and valve-fastening tool; b) Pre-operative subject-specific model displaying LV, LA, and RAV surfaces registered to the intra-operative space for visualization and navigation enhancement (note alignment of ventricular septum)

pre-operatively defined aortic valve annulus (AVA) and MVA with those identified intra-operatively, using a two-stage approach. First, we determined unit vectors normal to the pre- and intra-operative AVA and MVA. An initial alignment between the pre-operative and intra-operative annuli was obtained by minimizing the distance between both their centroids, as well as the tip of their corresponding unit vectors. In addition, the downhill simplex optimizer [13] was used to further minimize the distance between the two sets of annuli. Although the registration targets (AVA and MVA) are remote from the majority of the cardiac anatomy, this method nevertheless registers the pre-operative model to the patient in a manner sufficient to provide anatomical context for interpreting the US image.

\section{Conclusions}

In this paper we showed that accurate, pre-operative, dynamic representations of the surgical targets and surrounding anatomy can be generated and imported into our virtual surgical environment. We have demonstrated that our modelbased segmentation approach can successfully extract subject-specific dynamic representations of the mitral valve annulus throughout the cardiac cycle with a $3.1 \mathrm{~mm}$ accuracy.

As part of our future research, we plan to include a larger number of subjects in this study, and to ultimately extend this work towards in vivo animal studies, to show how employing models to augment the intra-operative VR environment can enhance surgical navigation.

Acknowledgments. The authors thank Dr. Daniel Bainbridge and Dr. Doug Jones for clinical consultation, and Dr. Usaf Aladl, Louis Estey, and Chris Wedlake for technical support. We also acknowledge funding for this work provided by the following Canadian agencies: NSERC, CIHR, ORDCF, OIT and CFI. 


\section{References}

1. Kypson, A.P., Felger, J.E., Nifong, L.W., Chitwood, W.R.: Robotics in valvular surgery: 2003 and beyond. Curr. Opin. Cardiol. 19, 128-133 (2004)

2. Vahanian, A., Acar, C.: Percutaneaous valve procedures: what is the future? Curr. Opin. Cardiol. 20, 100-106 (2005)

3. Edmunds, L.H.: Why cardiopulmonary bypass makes patients sick: strategies to control the blood-synthetic surface interface. Adv. Card. Surg. 6, 131-167 (1995)

4. McVeigh, E.R., Guttman, M.A., Lederman, R.J., Li, M., Hunt, T., Kozlov, S., Horvath, K.A.: Real-time interactive MRI-guided cardiac surgery: Aortic valve replacement using a direct apical approach. Magn. Reson. Med. 56, 958-964 (2006)

5. Suematsu, Y., Marx, G.R., Stoll, J.A., Dupont, P.E., Cleveland, R.O., Howe, R.D., Triedman, J.K., Mihaljevic, T., Mora, B.N., Savord, B.J., Salgo, I.S., del Nido, P.J.: Three-dimensional echo-guided beating-heart surgery without cardiopulmonary bypass: a feasibility study. J. Thorac. Cardiovasc. Surg. 128, 579-587 (2004)

6. Guiraudon, G.M.: Universal cardiac introducer. Patent Application, US 2005/0137609 A1, Appl. No. 10/736,786 (2005)

7. Vosburgh, K.G., San José Estépar, R.: Natural orifice transluminal endoscopic surgery (notes): An opportunity for augmented reality guidance. In: Stud Health Technol. Inform., vol. 125, pp. 485-490 (2007)

8. Linte, C.A., Wiles, A.D., Moore, J., Wedlake, C., Guiraudon, G.M., Jones, D.L., Bainbridge, D., Peters, T.M.: An augmented reality environment for imageguidance of off-pump mitral valve implantation. In: SPIE. Medical Imaging: Visualization and Image-Guided Procedures, vol. 6509, pp. 65090N-12 (2007)

9. Sauer, F.: Image registration: Enabling technology for image-guided surgery and therapy. In: IEEE, 27th Annual Conference of the Engineering in Medicine and Biology Society — EMBS, pp. 7242-7245. IEEE Computer Society Press, Los Alamitos (2005)

10. Wierzbicki, M.: Subject-specific models of the heart from 4D images. PhD Dissertation, Medical Biophysics, The University of Western Ontario, Canada (2006)

11. Moore, J., Drangova, M., Wierzbicki, M., Barron, J., Peters, T.M.: A highresolution dynamic heart model based on averaged MRI data. In: Ellis, R.E., Peters, T.M. (eds.) MICCAI 2003. LNCS, vol. 2878, pp. 549-555. Springer, Heidelberg (2003)

12. Lorenzo-Valdés, M., Sanchez-Ortiz, G.I., Mohiaddin, D., Rueckert, D.: Atlas-based segmentation and tracking of $3 \mathrm{D}$ cardiac $\mathrm{mr}$ images using non-rigid registration. In: Dohi, T., Kikinis, R. (eds.) MICCAI 2002. LNCS, vol. 2488, pp. 642-650. Springer, Heidelberg (2002)

13. Wierzbicki, M., Drangova, M., Guiraudon, G.M., Peters, T.M.: Validation of dynamic heart models obtained using non-linear registration for virtual reality training, planning, and guidance of minimally invasive cardiac surgeries. Med. Image. Anal. 8, 387-401 (2004)

14. Wiles, A.D., Guiraudon, G.M., Moore, J., Wedlake, C., Linte, C.A., Jones, D.L., Bainbridge, D., Peters, T.M.: Navigation accuracy for an intracardiac procedure using virtual reality-enhanced ultrasound. In: SPIE. Medical Imaging: Visualization and Image-Guided Procedures, vol. 6509, pp. 61410W-10 (2007) 